\title{
Innovaciones organizacionales y su efecto sobre el desempeño empresarial
}

\author{
Afcha Chávez, Sergio M.*
}

\section{Resumen}

A diferencia de las innovaciones tecnológicas la literatura que analiza la adopción y los efectos de las innovaciones organizacionales es relativamente escasa. Este hecho, obedece a las dificultades de conceptualización y medición de este concepto. Utilizando los datos del Panel de Innovación Tecnológica (PITEC) se analizan los factores que influyen en la adopción de innovaciones organizacionales, así como su efecto sobre distintas variables del desempeño empresarial, para ello se estima un modelo probit trivariante, mediante la aplicación del método máxima verosimilitud simulado, el cual, incorpora la simulación de Geweke-Hajivassiliou-Keane (GHK). Los resultados ponen de manifiesto la interdependencia entre los distintos tipos de innovaciones organizacionales y la necesidad de controlar esta interacción. Por otra parte, el análisis del efecto de las innovaciones organizacionales, permite concluir que aquellas relacionadas con la organización del trabajo son las que generan un mayor impacto sobre el desempeño empresarial.

Palabras clave: Innovación, innovaciones organizacionales, innovaciones administrativas, desempeño empresarial.

\section{Organizational Innovations and Their Effect on Business Performance}

\begin{abstract}
In contrast with the literature about technological innovations, literature analyzing the adoption and effects of organizational innovations is relatively scarce. This is due to the difficulties in conceptualizing and measuring this concept. Utilizing data from the Technological Innovation Panel (Panel de Innovación Tecnológica, PITEC, in Spanish), the factors that influence the adoption of organizational innovations as well as their effect on different variables for business performance were analyzed. To accomplish this, a trivariate probit model was estimated, through application of the maximum simu-
\end{abstract}

Recibido: 23-05-11 Aceptado: 04-10-11

* Economista (Universidad de Carabobo), Magister en Economía (Universidad de Barcelona). Docente investigador del Departamento de Economía Pública, Economía Política y Economía de la Universidad de Barcelona/España. e-mail: s.afcha@ub.edu 
lated verisimilitude model, incorporating the Geweke-Hajivassiliou-Keane (GHK) simulation. Results reveal interdependence between the different types of organizational innovations and the need to control this interaction. On the other hand, analysis of the effect of organizational innovations makes it possible to conclude that those related to organizing work generate a greater impact on business performance.

Key words: Innovation, organizational innovations, administrative innovations, business performance.

\section{Introducción}

Desde un punto de vista amplio se denomina innovación organizacional (IO en lo sucesivo) a todos aquellos cambios orientados a la mejora de los procesos existentes en el seno de una estructura organizacional. Dichos cambios implican la adopción de una idea o conducta nueva para la organización, y son adoptados por la empresa a fin de favorecer su desempeño y rentabilidad (Daft, 1978; Damanpour y Evan, 1984; Birkinshaw et al, 2008)

Las organizaciones adoptan innovaciones como respuestas a cambios en el conocimiento gerencial y tecnológico, la competencia industrial, expectativas futuras de directivos y accionistas sobre la mejora de su nivel de desempeño (Damanpour et al, 2009). La percepción de estos cambios y la forma de asimilarlos puede variar de una empresa a otra, y en consecuencia, los patrones de adopción de innovaciones pueden no resultar homogéneos. Estos patrones admiten diferencias a nivel industrial (manufactura o servicios), e incluso, como comentan Frambach y Schillewaert (2002) divergencias en las pautas de no adopción, cuyas variables determinantes pueden no ser el reflejo opuesto de aquellas que determinan la adopción de innovaciones organizacionales.

La literatura sobre IO intenta explicar los patrones de adopción de éste tipo de procesos por parte de las organizaciones. Una parte importante de estos estudios busca identificar las características principales de la estructura organizacional de las empresas innovadoras y analizar sus efectos sobre el desempeño de la empresa.

Sin embargo, los problemas de conceptualización y medición han dificultado la incorporación de un concepto operativo consensuado de IO. Esta situación ha generado que gran parte de la literatura en relación a este tema utilice conceptos distintos para referirse a estas innovaciones, con lo cual, la posibilidad de establecer comparaciones y extender las conclusiones obtenidas en algunos estudios a un plano más general, resulta complicado.

En un esfuerzo por estandarizar la medición y cuantificación de las IO la Oficina Estadística de la Comisión Europea (EUROSTAT, 2005) incorpora a partir del 2001 al cuestionario de la Encuesta Europea de Innovación (CIS) preguntas relacionadas con la introducción de las 10 y sus efectos ${ }^{1}$. En la actualidad la defini-

1 Para una descripción detallada de la evolución de la integración de este concepto en la CIS, véase Armbruster et al (2008). 
Innovaciones organizacionales y su efecto sobre el desempeño empresarial Afcha Chávez, Sergio $M$.

ción de IO utilizada en la CIS está basada en la propuesta por el Manual de Oslo (OCDE, 2005), lo cual, ha permitido el seguimiento y medición de tres tipos diferentes de innovaciones organizacionales claramente definidas.

Los datos utilizados en este trabajo corresponden al Panel de Innovación Tecnológica (PITEC) (FECYT, 2011). Esta base de datos constituye una rica fuente de información respecto a las actividades de innovación realizadas por las empresas del sector manufacturero y de servicios en España. Una de sus principales ventajas es que replica para España el cuestionario de la CIS con carácter anual, proporcionando una amplia base de datos que facilita el análisis empírico de la conducta empresarial con respecto a la implementación de innovaciones no tecnológicas.

Con base en esta información, la contribución principal de este trabajo consiste en identificar los determinantes de la implementación de nuevos métodos organizativos en tres ámbitos: i) el funcionamiento interno de la empresa (incluyendo métodos/sistemas de gestión del conocimiento); ii) en la organización del lugar de trabajo y iii) en las relaciones externas que no han sido utilizadas previamente por la empresa. Asimismo, se examina el efecto sobre el desempeño de la empresa en función de su propia valoración respecto a cuatro categorías específicas de desempeño organizacional. Se trata de un tema hasta ahora inexplorado en la literatura que permitirá avanzar en la comprensión de los mecanismos que subyacen a la incorporación de $\mathrm{IO}$.

\section{Innovaciones organizacionales: determinantes de su adopción y evidencia empírica relacionada}

En un libro clásico para comprender la adopción de las innovaciones y el cambio tecnológico Rosenberg (1993:75) señala con relación a los cambios sufridos por el sector de la construcción en la economía estadounidense durante la primera mitad del siglo $\mathrm{XX}$, que los cambios que permitieron el gran crecimiento de este sector no fueron fruto de grandes innovaciones de carácter técnico sino, más bien, de cambios menores de carácter acumulativo y, sobre todo, de cambios significativos en el ámbito organizativo. De esta manera, el autor señala como elementos determinantes de la productividad del sector los métodos de dirección y organización del proceso de la construcción.

Trabajos empíricos más recientes como el de Black y Lynch (2004) refuerzan el argumento anterior, señalando que alrededor del $30 \%$ de las ganancias de productividad del sector manufacturero en los Estados Unidos durante los años 90 es atribuible a factores relacionados con innovaciones de carácter organizacional. Dicho trabajo, hace referencia a prácticas innovadoras en la gestión de recursos humanos, tales como: procesos de reingeniería, nivel de participación de los empleados en la toma de decisiones, equipos auto-gestionados, esquemas de retribución basados en la participación en el reparto de beneficios, formación en el puesto de trabajo y uso de ordenadores entre los empleados con puestos no gerenciales. 
Estos resultados muestran una estrecha relación entre productividad e $1 \mathrm{O}$ y, sugieren que su adopción no solo genera una gestión más eficiente de los recursos humanos, cambios en la estructura de incentivos de los trabajadores o la optimización de los recursos empleados en la producción sino, también, que aquellas empresas que adoptan estas innovaciones pueden adaptarse con mayor facilidad a cambios externos e internos, potenciando de esta forma su capacidad de innovación tecnológica.

En general, la adopción de innovaciones es concebida como un mecanismo que permite a la empresa una conducta adaptativa generando cambios en la organización a fin de mantener o mejorar su desempeño. Realizar actividades innovadoras permite a las organizaciones que introducen cambios tempranos a ser conscientes de los últimos desarrollos, absorber conocimientos nuevos e incrementar sus oportunidades de beneficiarse de las actividades innovadoras (Cohen y Levinthal, 1990; Roberts y Amit, 2003).

Varios factores motivan la adopción de innovaciones organizativas. Las oportunidades, amenazas y cambios en el entorno conducen a las empresas a generar cambios adaptativos. La introducción de innovaciones en general y de 10 en particular, permite a las empresas reconfigurar sus funciones internas y externas para dar respuesta a las demandas de su entorno, operar eficientemente y mantener o mejorar su desempeño. Dichos cambios también obedecen a la percepción de los directivos de la empresa respecto a posibles situaciones en el entorno de la empresa que comprometan su capacidad para actuar de forma efectiva ó cuando existen expectativas de nuevas oportunidades que pueden ser aprovechadas y/o nuevos productos y servicios que pueden contribuir al desempeño de la empresa (Wischnevsky y Damanpour, 2006).

Muchas de las teorías organizacionales, conciben las organizaciones como sistemas abiertos y adaptativos en las que se introducen cambios para mantener sus objetivos y garantizar un buen funcionamiento a lo largo del tiempo (Damanpour et al, 2009). Por ejemplo, Pfeffer y Salancik (2003) enfatizan la importancia de la elección del modelo de gerencia que permite a la empresa gestionar las interacciones entre la organización y su entorno en respuesta a los distintos condicionantes externos como la escasez de recursos, la demanda de los clientes, proveedores y acreedores.

En consistencia con lo anterior, las organizaciones innovan para obtener ventaja sobre sus competidores mediante una reacción anticipada (Geroski, 1995; Roberts y Amit, 2003). La importancia de las IO en la competitividad ha sido confirmada en varios trabajos que analizan su impacto sobre el desempeño de las empresas (Caroli y Van Reenen, 2001; Damanpour y Evan, 1984; Greenan 2003; Piva et al, 2006).

Estas investigaciones ponen de manifiesto dos puntos diferentes. Primero, que las 10 actúan como prerrequisito en la asimilación del cambio técnico y, por consiguiente, tienen un efecto facilitador de las innovaciones tecnológicas de proceso y de producto. Este fenómeno obedece a que la generación de innovaciones tecnológicas está condicionada por la capaci- 
Innovaciones organizacionales y su efecto sobre el desempeño empresarial Afcha Chávez, Sergio $M$.

dad organizacional de la empresa para integrar nueva tecnología.

En segundo lugar, la literatura pone de manifiesto que las 10 presentan una fuente de ventajas competitivas ya que ellas mismas tienen un impacto significativo sobre el desempeño de la empresa en relación a la productividad, plazos de entrega, calidad y flexibilidad (Goldman et al, 1995; Hammer y Champy, 1995; Womack et al, 1990). A su vez, determinados tipos de innovación, favorecen el desempeño financiero de la empresa, especialmente en entornos dinámicos y competitivos (Jansen et al, 2006).

La literatura sobre innovación organizacional se ha visto sin embargo limitada por las dificultades que representan su conceptualización y medición. La mejora en la disponibilidad de datos respecto a la medición de las innovaciones no tecnológicas o socio-tecnológicas, representa una oportunidad para avanzar en la comprensión de los patrones de adopción de innovaciones organizacionales seguidos por la empresa y sus efectos al ser combinadas con otro tipo de innovaciones (Armbruster et al, 2008). Polder et al (2010) examinan la complementariedad de las IO y las innovaciones de producto y proceso en función de su inversión en tecnologías de la información y comunicaciones (TICs). Sus resultados reportan un efecto positivo sobre la productividad asociado a la incorporación conjunta de innovaciones tecnológicas y la adopción de cambios organizacionales. Al igual que Gera y Gu (2004) revelan que la inversión en TICs funciona como elemento integrador de dichas innovaciones y, que su introducción combinada junto con innovaciones organizacionales genera un efecto positivo sobre el desempeño de las empresas.

El efecto de las IO sobre la organización facilita la incorporación de nuevo conocimiento y funciona como marco para la generación de innovaciones de carácter técnico. En consecuencia, la adopción de cambios organizacionales es concebida como un prerrequisito para la generación de innovaciones tecnológicas (Lam 2004).

Esto es confirmado en trabajos empíricos recientes, donde se muestra que la incorporación conjunta de innovaciones tecnológicas y organizacionales, tienen un efecto complementario sobre la productividad y el desempeño de la empresa (Damanpour et al, 2009; Polder et al, 2010).

Es posible, por tanto, distinguir entre un efecto inmediato, asociado a las ventajas que la propia IO genera en la asignación de recursos dentro de la empresa y un efecto indirecto que se desprende del rol de la IO como elemento potenciador de otras innovaciones y de la gestión de nuevo conocimiento a nivel interno.

Dumanpour (2009) y Subramanian y Nilakanta (1996) confirman los efectos positivos asociados a la combinación de diferentes tipos de innovación en las organizaciones. Este efecto es significativo especialmente en el caso del sector servicios, donde las innovaciones son principalmente incrementales y la empresa se beneficia primeramente de innovar en varios ámbitos e interactuar con sus clientes, proveedores, socios y accionistas. 


\section{Innovaciones organizacionales: un modelo estadístico}

Los sucesivos avances en la medición de aspectos socio-tecnológicos se han reflejado en la Encuesta Europea de Innovación (CIS) a partir de la incorporación en 2001 de campos relacionados con las innovaciones organizacionales. La variable dependiente utilizada en esta investigación, hace referencia precisamente a la introducción de innovaciones organizacionales y su efecto en la organización. Los años para los que se encuentra disponible esta información son el 2004 y el 2005.

Los datos utilizados corresponden al Panel de Innovación Tecnológica (PITEC). Esta encuesta aplicada con carácter anual desde 2001, replica para España el cuestionario utilizado por la CIS. De esta forma, las preguntas utilizadas en el PITEC coinciden con las distintas oleadas de la CIS.

El cuestionario aplicado en las ediciones 2004 y 2005 del PITEC define las IO como la implementación de nuevos métodos organizativos en tres ámbitos: i) el funcionamiento interno de la empresa (incluyendo métodos/sistemas de gestión del conocimiento); ii) en la organización del lugar de trabajo; y iii) en las relaciones externas que no han sido utilizadas previamente por la empresa. Dicha implementación debe ser el resultado de decisiones estratégicas llevadas a cabo por la dirección de la empresa. Excluye fusiones y adquisiciones aunque estas supongan una novedad organizativa para la empresa.
A partir de la inclusión de estos ítems en la encuesta, las empresas declaran si adoptaron o no alguno de estos tres tipos de innovación organizacional. Esta información podría estar censurada en la medida que las empresas no reporten la introducción de innovaciones. Tal y como señalan Polder et al (2010) esto puede ocurrir, por ejemplo, en el caso que los empleados lleven a cabo esta actividad de manera informal, o bien, si la innovación organizacional no es reconocida como tal por los empleados de la empresa.

Para estimar adecuadamente el efecto de las innovación organizacional sobre el desempeño organizacional es necesario, por tanto, eliminar dicho sesgo de selección. Sin embargo, la decisión respecto al tipo de innovación organizacional emprendida no parece una decisión independiente sino, por el contrario, una decisión que se realiza de manera simultánea. La aplicación de modelos univariantes para determinar la probabilidad de introducir una innovación organizacional de alguno de los tres tipos señalados anteriormente, no sería adecuada para el análisis de este tipo de situaciones, dado que podría estar correlacionada.

Para solventar este problema se propone la estimación de un modelo probit trivariante que permite la estimación simultánea de tres variables dependientes dicotómicas. Este modelo contempla de forma explícita la existencia de autocorrelación en el término de error y, por tanto, permite controlar el sesgo ocasionado por la existencia de correlación entre variables no observables que determinan la adopción de las distintas innovaciones. La estimación de este modelo se realiza mediante la aplicación del método 
Innovaciones organizacionales y su efecto sobre el desempeño empresarial Afcha Chávez, Sergio M.

máxima verosimilitud simulado, el cual, incorpora la simulación de Geweke-Hajivassiliou-Keane $(\mathrm{GHK})^{2}$.

El modelo probit trivariante que permite identificar las variables que influyen sobre la introducción de innovaciones organizacionales está determinado por:

$O I_{1}^{*}=\beta_{11} x+\varepsilon_{1} O I=1$ si OI $I_{1}^{*}>0$, Odeotraforma

$O I_{2}^{*}=\beta_{21} x+\varepsilon_{2} O I=1 \mathrm{siO} I_{2}^{*}>0$, 0de otraforma

$O I_{3}^{*}=\beta_{31} x+\varepsilon_{31} \mathrm{OI}=1$ siOI ${ }_{3}^{*}>0$, Ode otraforma

Donde $O I_{1}^{*}, O I_{2}^{*}$ y $O I_{3}^{*}$ representan los verdaderos valores no observados de la propensión a introducir innovaciones organizacionales relacionadas con: i) la introducción de nuevos sistemas de gestión del conocimiento; ii) la organización y gestión de recursos humanos; y iii) innovaciones asociadas a las relaciones externas de la empresa. $\mathrm{OI}_{1} \mathrm{Ol}_{2} \mathrm{y} \mathrm{OI}_{3}$ son las variables dicotómicas observadas que indican si la empresa introdujo alguno de los tipos de 10 mencionados anteriormente, $X$ es un vector de variables que influyen sobre la probabilidad de introducir algún tipo de innovación y $\varepsilon$, el término de error aleatorio.

A partir de la estimación de este modelo probit es posible calcular las predicciones de la probabilidad de introducir una innovación organizacional del tipo 1 , 2 o 3, e incorporarla para determinar el efecto de las innovaciones organizacionales. El resultado de esta primera estimación permitirá, por un lado, cumplir con el primer objetivo planteado de identificar los determinantes de los tres tipos de innovación organizacional y, por otro lado, resolver el problema de selección muestral planteado en un inicio.

El paso siguiente consiste en estimar el efecto de introducir estas innovaciones organizacionales sobre cuatro tipos de desempeño organizacional: i) Tiempos de respuesta de la empresa hacia sus clientes y proveedores; ii) Costes por unidad producida; iii) Calidad de los bienes y servicios producidos; iv) Satisfacción del personal empleado. Estos indicadores son valorados según una escala tipo Likert de 1 al 4 en función de si la innovación organizacional tuvo un impacto elevado, intermedio, reducido o no pertinente. Los valores de la variable resultado son de carácter ordinal y, en consecuencia, no es posible establecer la distancia cardinal entre cada uno de los posibles resultados. La variable de interés $\mathrm{Y}$ toma $\mathrm{H}$ categorías de respuestas $y h$, donde $h=1, \ldots, \mathrm{H}$ ordenadas de la siguiente manera:

$$
y_{1}<y_{2}<\ldots<y_{H}
$$

Siguiendo a Miranda y RabeHesketh (2006) la variable respuesta latente $\mathrm{xx}$, para el iesimo individuo, es determinada de acuerdo a la siguiente ecuación:

$y_{i}^{*}=x_{i}^{*} \beta+\lambda \varepsilon_{i}+\tau_{i}$

Donde, $x_{i}$ representa un vector de variables explicativas y $\lambda, \varepsilon_{t}$ y $\tau_{i}$ representan un factor de carga, un término de heterogeneidad y el error aleatorio, respectivamente. El término constante es sustituido por umbrales que determinan el 
cambio de una categoría a otra de las respuestas observadas:

$$
y_{i}\left\{\begin{array}{l}
1 \text { si }-\infty<y_{i}^{*}<k_{1} \\
2 \text { si } k_{1}<y_{i}^{*}<k_{2} \\
3 \text { si } k_{2}<y_{i}^{*}<k_{3} \\
4 \text { si } k_{2}<y_{i}^{*}<k \infty
\end{array}\right.
$$

Donde, $\mathrm{k}_{\mathrm{s}} \mathrm{S}=1 \ldots \mathrm{H}-1$ representa el parámetro correspondiente a los distintos umbrales. Esta es una variable latente que solo se hace observable para aquellas empresas que declaran haber obtenido una innovación organizacional y asignan una valoración sobre el efecto logrado.

\subsection{Descripción de las variables que influyen en la adopción de innovaciones organizacionales}

A continuación se enumeran las variables utilizadas para estimar la regresión que identifica los factores que afectan la adopción de los diferentes tipos de innovación organizacional. El Cuadro 1 muestra estas variables clasificadas en torno a cuatro dimensiones. En primer lugar, se incluye un conjunto de variables que indican las actividades innovadoras llevadas a cabo por la empresa para conseguir nuevos productos basados en la aplicación de ciencia y tecnología.

\section{Cuadro 1}

Variables independientes que afectan la adopción de innovaciones organizacionales.

\begin{tabular}{cl}
\hline Factores tecnológicos & Adquisición de I+D (I+D externa) \\
& Adquisición de maquinaria de software-hardware \\
& Adquisición de otros conocimientos externos de innovación \\
& (no incluido en I+D) \\
& Introducción de innovaciones en el mercado \\
& Diseños y otros preparativos \\
& Cooperación tecnológica \\
\hline \multirow{2}{*}{ Obstáculos a la innovación } & Falta de fondos de la empresa \\
& Falta de financiación por fuentes externas \\
& Costes de innovación elevados \\
& Falta de personal cualificado \\
& Falta de información sobre tecnología \\
& Falta de información sobre mercados \\
& Dificultad para encontrar socios. \\
\hline Recursos humanos & Formación \\
& Formación en el período anterior \\
& $N^{0}$ investigadores en I+D \\
& $N^{0}$ técnicos en I+D \\
& $N^{0}$ personas auxiliares en I+D \\
\hline Financiación & Fondos propios \\
& Fondos públicos \\
\hline
\end{tabular}

Fuente: Elaboración propia. 
Innovaciones organizacionales y su efecto sobre el desempeño empresarial Afcha Chávez, Sergio $M$.

Como se ha remarcado, las innovaciones organizacionales permiten potenciar otras innovaciones de la empresa, con lo cual, las innovaciones organizacionales pueden ser también concebidas como una vía para superar parte de los obstáculos a los que se enfrentan las empresas para poder innovar. Es por ello que se han incorporado las variables que indican si la empresa presenta determinadas barreras para innovar.

El tamaño de la empresa ha sido señalado en trabajos empíricos como un factor determinante en la adopción de innovaciones organizacionales. Se espera que las empresas de mayor tamaño tengan los recursos necesarios para emprender innovaciones organizacionales y que, por otro lado, la necesidad por mantener una estructura organizacional adecuada a sus dimensiones genera una presión adicional en las empresas de mayor dimensión. Así mismo, la incorporación de innovaciones organizacionales requiere fundamentalmente de trabajadores cualificados para emprender cambios a nivel interno. Para medir la influencia de este factor se tienen en cuenta el número de empleados dedicados a actividades de I+D según su titulación.

Por último, se incluyen dos variables relacionadas con la capacidad financiera de la empresa para emprender innovaciones organizacionales. Para ello, se utiliza el porcentaje de fondos propios de la empresa y el porcentaje de fondos públicos recibidos para emprender actividades de carácter innovador.

\subsection{Descripción de las variables que afectan sobre el desempeño de la empresa}

La encuesta del PITEC proporciona la valoración de la empresa en referencia a cuatro dimensiones de desempeño organizacional: i) Reducción del periodo de respuesta a las necesidades de un cliente o proveedor; ii) Mayor calidad de los bienes y servicios; iii) Menores costes por unidad producida; iv) Mejora en la satisfacción del personal o disminución de las tasas de rotación del mismo. El objetivo principal de este trabajo es determinar cómo influye la adopción de IO sobre esta valoración. A fin de controlar el resto de factores que también pueden influir sobre esta valoración, se incorporan las siguientes variables:

Tamaño de la organización: Esta variable es empleada con frecuencia para explicar la adopción de innovaciones en el seno de las organizaciones. Su influencia resulta ambigua ya que, por un lado, el tamaño de la empresa influye positivamente sobre las necesidades de mejora y soporte del desempeño organizacional. Trabajos como el de Lynch (2007), asocian la adopción de innovaciones organizativas a la presencia de beneficios de años anteriores y el buen desempeño financiero de la empresa, elementos que con frecuencia resultan beneficiados por el tamaño de la empresa. Sin embargo, otros trabajos argumentan que las empresas pequeñas al poseer estructuras organizacionales más flexibles favorecen la adopción de innovaciones y 
la adaptación del cambio tecnológico. El número de empleados y la cifra de negocios son utilizados como indicadores del tamaño de la empresa.

Clase de empresa: Indica si la empresa es de capital privado, público o privado-internacional y si pertenece a un grupo de empresas.

Nivel de innovación de la empresa: La propensión de la empresa para adoptar nuevos productos o ideas puede influir en la adopción de innovaciones organizacionales (Lynch, 2007). Para tener en cuenta el efecto de la capacidad innovadora en la adopción de innovaciones organizacionales, se tiene en cuenta si la empresa ha introducido bienes o servicios en el mercado.

Cooperación tecnológica: Esta variable indica el nivel de contacto de la empresa con otras instituciones y agentes del sistema. En la medida que la empresa está conectada con empresas proveedoras, clientes, universidades, centros tecnológicos o empresas competidoras, la propensión a adoptar innovaciones organizacionales aumenta. La literatura empírica muestra que las empresas que mantienen acuerdos de cooperación con empresas o instituciones públicas de investigación científica tienen un mejor desempeño innovador (Laursen y Salter, 2004; Afcha, 2011). En este apartado se utiliza la variable de cooperación con otras empresas o instituciones.

Apertura al exterior: Las empresas cuyo porcentaje de ventas en el extranjero es elevado suelen tener un mayor nivel de innovación, al estar sometido a presiones competitivas más fuertes. Como variables indicadoras de esta cate- goría se incluye el porcentaje de exportaciones de la empresa y la participación de capital extranjero en la empresa.

Capital humano de la empresa: Entre las formas de innovación organizacional se contempla la implantación de nuevas técnicas de recursos humanos, el empoderamiento de los trabajadores, la descentralización de la toma de decisiones, rotación de puestos de trabajo, esquemas de flexibilidad laboral y otras formas de gerencia de recursos humanos que requieren de una formación previa del personal de la empresa.

En la medida que la empresa cuente con personal cualificado para la realización de sus actividades productivas, tendrá mayor posibilidad de incorporar nuevas formas de gestión que impliquen una mayor responsabilidad a sus trabajadores o, una mayor autonomía e independencia en la organización laboral. Se utiliza dentro de esta categoría las siguientes variables: el número total de trabajadores vinculados a actividades de I+D y la formación de trabajadores

Financiación de las actividades de I+D de la empresa: Lynch (2007) indica que la obtención de beneficios futuros actúa como determinante en la adopción de innovaciones organizacionales, poniendo de relieve que las restricciones financieras a las que se enfrentan las empresas pueden condicionar la introducción de este tipo de innovaciones. Se incluye la variable fondos propios para evaluar si las empresas de mayor autonomía financiera generan innovaciones de mayor impacto en alguno de los ámbitos de desempeño organizacional analizado. 
Innovaciones organizacionales y su efecto sobre el desempeño empresarial Afcha Chávez, Sergio $M$.

\section{Innovaciones organizacionales y desempeño empresarial}

La Tabla 1 muestra los resultados de la estimación probit trivariante. En primer lugar, destaca la significancia estadística de los coeficientes de correlación entre los distintos tipos de innovaciones organizacionales. Este resultado confirma la necesidad de adoptar una aproximación multivariante para obtener una correcta predicción de la probabilidad de introducir innovaciones organizacionales.

La existencia de elementos comunes en las empresas que adoptan los distintos tipos de innovación organizacional podría ser una explicación a esta interdependencia. En este sentido, los resultados sitúan la capacidad de adquisición de conocimiento como un elemento que actúa de forma transversal en la adopción de distintas innovaciones organizacionales (Chang y Lee, 2008). En nuestro caso, los resultados obtenidos permiten identificar precisamente tres variables que afectan positivamente la adopción de los tres tipos de innovaciones organizacionales, se trata de las variables relacionadas con la cooperación, las actividades formativas de los empleados en el periodo corriente y las actividades formativas desarrollados en el periodo t-1. Estas variables afectan claramente la capacidad de adquisición de conocimiento de la empresa, confirmando, en cierta forma, que en aquellas empresas que refuerzan su capacidad de asimilación de nuevo conocimiento, la introducción de $1 O$ tienen un carácter complementario, es decir, la adopción de una de ellas, influye positivamente en la propensión a adoptar otro tipo distinto de innovación organizacional.

En un sentido similar Polder (2010) y Damanpour (2009) analizan la interacción entre innovaciones no tecnológicas (i.e. innovaciones organizacionales) e innovaciones tecnológicas, confirmando que en presencia de innovaciones organizacionales, la introducción de innovaciones de carácter tecnológico tienen un mayor efecto sobre el desempeño de la empresa.

Las dos primeras columnas muestran los resultados respecto a la introducción de nuevos sistemas de gestión del conocimiento, $\mathrm{IO}_{1}$. Este tipo de innovaciones se ven positivamente afectada por aquellos factores relacionados con los flujos de información de la empresa. Esto queda parcialmente confirmado por la influencia positiva que ejerce el tamaño de la empresa sobre la introducción de nuevos sistemas de conocimiento, ya que, por lo general, las empresas de mayor dimensión manejan flujos de información de mayor magnitud, aumentando con ello la complejidad en su gestión y correcta asimilación. Otro elemento que influye significativamente en la adopción de nuevos sistemas de gestión del conocimiento es la introducción de TICs. La significancia estadística de la variable de adquisición de maquinaria, software y hardware, confirma la importancia de estas tecnologías en la implementación de innovaciones organizacionales. La relevancia de las TICs en la implementación de innovaciones organizacionales ha quedado patente en los trabajos de Polder et al (2010) y Gera y Gu (2004). 
Tabla 1 Determinantes de la introducción de innovaciones organizacionales.

\begin{tabular}{|c|c|c|c|c|c|c|}
\hline & \multicolumn{2}{|c|}{ Innov. Org. 1} & \multicolumn{2}{|c|}{ Innov. Org. 2} & \multicolumn{2}{|c|}{ Innov. Org. 3} \\
\hline & Coef. & Std. Err. & Coef. & Std. Err. & Coef. & Std. Err. \\
\hline $\mathrm{N}^{\circ}$ de trabajadores totales & 0,000 & $0,000^{\star * *}$ & 0,000 & $0,000^{* *}$ & 0,000 & 0,000 \\
\hline Falta de fondos empresa & $-0,035$ & 0,036 & 0,003 & 0,036 & 0,044 & 0,040 \\
\hline $\begin{array}{l}\text { Falta de financiación (Fuentes } \\
\text { externas) }\end{array}$ & 0,018 & 0,035 & 0,026 & 0,035 & 0,001 & 0,038 \\
\hline Coste innovación elevado & $-0,033$ & 0,031 & $-0,021$ & 0,031 & $-0,051$ & 0,033 \\
\hline Falta personal cualificado & 0,095 & $0,039^{* * *}$ & 0,058 & 0,039 & 0,013 & 0,043 \\
\hline Falta información sobre tecnología & $-0,011$ & 0,047 & $-0,033$ & 0,046 & $-0,041$ & 0,051 \\
\hline Falta información mercados & 0,056 & 0,040 & 0,049 & 0,040 & 0,060 & 0,043 \\
\hline Dificultad para encontrar socios & $-0,006$ & 0,031 & 0,057 & $0,030^{* *}$ & 0,103 & $0,033^{* * *}$ \\
\hline I+D externa & 0,023 & 0,060 & 0,077 & 0,059 & 0,173 & $0,062^{\star * *}$ \\
\hline $\begin{array}{l}\text { Adq. } \\
\text { Maquinaria-software-hardware }\end{array}$ & 0,355 & $0,055^{* * *}$ & 0,239 & $0,054^{* * *}$ & 0,093 & 0,059 \\
\hline $\begin{array}{l}\text { Adq. conocimientos externos } \\
\text { innovación }\end{array}$ & $-0,024$ & 0,116 & 0,042 & 0,113 & 0,082 & 0,118 \\
\hline Formación & 0,339 & $0,071^{* * *}$ & 0,251 & $0,068^{* * *}$ & 0,179 & $0,072^{* * *}$ \\
\hline Formación en t-1 & 0,208 & $0,052^{* * *}$ & 0,200 & $0,052^{* * *}$ & 0,198 & $0,057^{* * *}$ \\
\hline Introd. Innov. Mercado & 0,077 & 0,063 & 0,083 & 0,061 & 0,106 & 0,065 \\
\hline Diseño y otros preparativos & 0,135 & 0,108 & 0,117 & 0,105 & 0,137 & 0,108 \\
\hline Cooperación & 0,187 & $0,055^{\star \star *}$ & 0,134 & $0,054^{* * *}$ & 0,401 & $0,058^{* * *}$ \\
\hline$N^{0}$ investigadores en $1+D$ & 0,000 & 0,002 & $-0,002$ & 0,002 & 0,001 & 0,001 \\
\hline$N^{0}$ técnicos en $1+D$ & 0,002 & 0,002 & 0,000 & 0,002 & 0,002 & $0,001^{* *}$ \\
\hline $\mathrm{N}^{\circ}$ de auxiliaries & $-0,002$ & 0,002 & $-0,004$ & 0,002 & $-0,002$ & 0,002 \\
\hline Fondos propios & 0,001 & 0,002 & 0,003 & 0,002 & $-0,001$ & 0,002 \\
\hline Fondos públicos & $-0,002$ & 0,002 & 0,001 & 0,002 & 0,001 & 0,001 \\
\hline Intercepto & $-0,566$ & $0,063^{* * *}$ & $-0,718$ & $0,063^{* * *}$ & $-1,3$ & $0,074^{* * *}$ \\
\hline \multicolumn{7}{|l|}{ Coeficientes de Correlación } \\
\hline Inn. Org. 2-Inn. Org.1 & 0,636 & $0,021^{* \star *}$ & & & & \\
\hline Inn. Org. 3- Inn. Org.1 & 0,474 & $0,028^{* * *}$ & & & & \\
\hline Inn. Org. 2-Inn. Org.2 & 0,530 & $0,026^{* * *}$ & & & & \\
\hline
\end{tabular}

Number of obs $=2691$

Wald chi2 $(63)=433,27$

Log $\rightarrow$ likelihood $=-4427.6538$ Prob $>$ chi $2=0,00$

Nota: Estadísticamente significativo al ${ }^{* * *} 99 \%,{ }^{* *} 95 \%$ y ${ }^{*} 90 \%$ de confianza.

Fuente: Elaboración propia. 
Innovaciones organizacionales y su efecto sobre el desempeño empresarial Afcha Chávez, Sergio $M$.

Las variables relacionadas con la cualificación de los recursos humanos también muestran una influencia significativa sobre la incorporación de este tipo de IO. Tanto la inversión en formación, como el reconocimiento por parte de la empresa de falta de personal cualificado para desarrollar adecuadamente sus innovaciones, muestran que las empresas que incorporan nuevos sistemas de gestión del conocimiento están interesadas en mejorar y superar las barreras originadas por el déficit en capital humano revelado por la empresa.

Por último, la cooperación con terceras partes también influye positivamente sobre la introducción de IO1. El establecimiento de este tipo de relaciones expresa un interés en la búsqueda de fuentes externas de conocimiento y, por tanto, implica un proceso de transferencia de información entre la empresa y sus socios, que hacen necesaria la mejora continua de los sistemas de gestión del conocimiento disponibles.

Las columnas 3 y 4 pertenecen a la estimación sobre las innovaciones organizacionales que implican nuevos métodos de organización en los lugares de trabajo. Estas innovaciones están asociadas a la mejora en el reparto de responsabilidades, el proceso de toma de decisiones o la gestión de sus equipos de trabajo. En este sentido, los resultados muestran que las empresas que invierten en la formación de sus trabajadores y en la adquisición de equipos que puedan conducir a una mayor productividad de dichos trabajadores (inversión en maquinaria, hardware y software), tienen una mayor probabilidad de adoptar nuevos métodos para la organización de los lugares de trabajo.

Nuevamente el tamaño de la empresa influye positivamente sobre la posibilidad de introducir una innovación de este tipo. Esto responde a una mayor necesidad por parte de las empresas de mayor dimensión por mantener un nivel de organización laboral y una estructura organizacional adecuada al tamaño de la empresa. Este resultado es confirmado por Fariñas y López (2011) quienes encuentran que la propensión a introducir innovaciones organizacionales del tipo 1 y 2 es creciente con el tamaño de la empresa, y en forma de $U$, para las innovaciones organizacionales de tipo 3.

Asimismo, es posible constatar que la existencia de acuerdos de cooperación tecnológica con socios externos induce a la empresa a buscar este tipo de innovaciones organizacionales, evidenciando que las empresas que mantienen este tipo de acuerdos cooperativos, necesitan una organización laboral a nivel interno que permita sacar el máximo provecho a los distintos fuentes de conocimiento utilizadas en el proceso de innovación.

En cuanto a las innovaciones organizacionales del tipo 3, mejora en los métodos de gestión de las relaciones externas, influye, tal y como cabía esperar, que las empresas realicen actividades externas de I+D y que mantengan actividades de cooperación tecnológica. Asimismo, la dificultad para encontrar socios influye positivamente en la introducción de innovaciones del tipo 3 , mostrando nuevamente la existencia de cierta relación entre la búsqueda de innovaciones organizacionales como respuesta a las dificultades presentes en el proceso de 
innovación. Al igual que en los casos de las $1 O_{1}$ y $1 O_{2}$, la formación de recursos humanos se revela como un factor determinante para conseguir mejoras en la gestión de las relaciones externas de la empresa.

Finalmente, la existencia de técnicos dedicados a actividades de I+D también influye positivamente en la adopción de métodos que contribuyen en la mejora de gestión de las relaciones externas.

La Tabla 2 muestra el efecto de introducir innovaciones organizacionales sobre distintos ámbitos del desempeño organizacional de la empresa. Para cada una de las categorías de desempeño organizacional evaluadas se estimó una regresión probit ordenado. Los coeficientes de dicha regresión pueden ser interpretados como la influencia de cada variable sobre el grado de repercusión observado en cada uno de los ámbitos de desempeño organizacional analizados.

Para una mejor interpretación de los resultados mostrados en la Tabla 2, se ha añadido una tercera columna que muestra los resultados reescalados y estandarizados, cuya interpretación permite una aproximación similar a la empleada en modelos lineales ${ }^{3}$.

El primero de los efectos analizados tiene que ver con la reducción de tiempos de respuesta a las necesidades de un cliente o proveedor. Cabe destacar en primer lugar el efecto de cada uno de los tipos de IO sobre la reducción de tiempos de respuesta. Mientras que la introducción de nuevos métodos de organiza- ción de los lugares de trabajo influye positivamente en la reducción de tiempos de respuesta, la introducción de nuevos sistemas de gestión del conocimiento y de nuevos métodos de gestión de relaciones externas, disminuyen la probabilidad de reducir dichos tiempos de respuesta.

Las distintas categorías de innovaciones de proceso: nuevos métodos de fabricación de bienes o servicios, nuevos sistemas logísticos o métodos de entrega o distribución y nuevas actividades de apoyo en procesos, como sistemas de mantenimiento, operaciones informáticas, compra o contabilidad, afectan positivamente sobre la repercusión que la introducción de innovaciones organizacionales tiene sobre la reducción de tiempos de entrega. Por último el mantenimiento de acuerdos cooperativos afecta positivamente en la reducción de los tiempos de espera.

El segundo modelo corresponde a los determinantes de la reducción de costes de producción. La columna 2, muestra que las variables tamaño de la empresa, pertinencia a un grupo y el hecho de ser una empresa privada y privada con participación internacional, generan un efecto positivo sobre la reducción de costes de producción. El que la introducción de innovaciones organizacionales tenga una mayor repercusión sobre empresas con este tipo de características, evidencia la aparición de economías de escala y de las sinergias que es posible conseguir en empresas de mayor dimensión, o pertenecientes a un grupo, donde la introduc-

3 Para una revisión detallada de este procedimiento véase Long y Freese (2001) 
Innovaciones organizacionales y su efecto sobre el desempeño empresarial Afcha Chávez, Sergio M.

Tabla 2

Efecto de las innovaciones organizacionales.

\begin{tabular}{|c|c|c|c|c|c|c|c|c|c|c|c|c|}
\hline & \multicolumn{2}{|c|}{$\begin{array}{l}\text { Reducción } \\
\text { Respuesta a } \\
\text { Proveedores }\end{array}$} & \multirow{2}{*}{$\begin{array}{c}\text { Tiempos } \\
\text { Clientes } \\
\text { Bstdxy }\end{array}$} & \multicolumn{3}{|c|}{$\begin{array}{c}\text { Costes } \\
\text { Producción }\end{array}$} & \multicolumn{3}{|c|}{ Calidad } & \multicolumn{3}{|c|}{$\begin{array}{c}\text { Satisfacción } \\
\text { de los empleados }\end{array}$} \\
\hline & Coef. & Std. Err. & & Coef. & Std. Err. & Bstdxy & Coef. & Std. Err. & Bstdxy & Coef. & Std. Err. & Bstdxy \\
\hline Start-up & 0,034 & 0,171 & 0,015 & $-0,161$ & 0,167 & $-0,025$ & $-0,499$ & $0,169^{\star * *}$ & $-0,078$ & 0,087 & 0,169 & 0,014 \\
\hline $\mathrm{N}^{\circ}$ de empleados & 0,000 & 0,000 & 0,015 & 0,000 & $0,00^{* *}$ & 0,058 & 0,000 & 0,000 & 0,028 & 0,000 & 0,000 & 0,009 \\
\hline $\begin{array}{l}\text { Pertenencia a un } \\
\text { grupo }\end{array}$ & $-0,040$ & 0,062 & 0,015 & 0,205 & $0,060^{\star \star \star}$ & 0,098 & $-0,087$ & 0,063 & $-0,042$ & $-0,007$ & 0,061 & $-0,003$ \\
\hline Empresa publica & 0,014 & 0,181 & 0,015 & 0,084 & 0,178 & 0,019 & $-0,071$ & 0,187 & $-0,016$ & $-0,217$ & 0,179 & $-0,049$ \\
\hline Empresa privada & 0,087 & 0,146 & 0,015 & 0,335 & $0,143^{*}$ & 0,127 & 0,097 & 0,151 & 0,037 & 0,006 & 0,144 & 0,002 \\
\hline $\begin{array}{l}\text { Privada } \\
\text { internacional }\end{array}$ & 0,175 & 0,176 & 0,015 & 0,505 & $0,173^{\star *}$ & 0,140 & 0,132 & 0,182 & 0,037 & 0,062 & 0,174 & 0,018 \\
\hline $\begin{array}{l}\text { Prob. } \\
\text { Introducir } 101\end{array}$ & $-2,236$ & $0,755^{\star \star \star}$ & 0,015 & $-3,390$ & $0,741^{* \star *}$ & $-0,483$ & $-1,690$ & $0,776^{* *}$ & $-0,242$ & $-2,434$ & $0,742^{* * *}$ & $-0,353$ \\
\hline $\begin{array}{l}\text { Prob } \\
\text { Introducir IO2 }\end{array}$ & 4,368 & $12,93^{\star \star \star}$ & 0,015 & 6,147 & $1,272^{\star \star \star}$ & 0,757 & 2,455 & $1,329^{*}$ & 0,304 & 3,329 & $1,270^{* * *}$ & 0,418 \\
\hline $\begin{array}{l}\text { Prob. } \\
\text { Introducir } 1 \mathrm{O} 3\end{array}$ & $-1,944$ & $0,827^{\star \star}$ & 0,015 & $-2,451$ & $0,811^{\text {***}}$ & $-0,320$ & $-0,780$ & 0,857 & $-0,103$ & $-0,726$ & 0,812 & $-0,097$ \\
\hline $\begin{array}{l}\text { Nuevo método de } \\
\text { fabricación }\end{array}$ & 0,127 & $0,060^{\star \star}$ & 0,015 & 0,091 & 0,059 & 0,043 & 0,267 & $0,062^{\star \star *}$ & 0,126 & 0,037 & 0,059 & 0,018 \\
\hline $\begin{array}{l}\text { Nuevos sistema } \\
\text { logístico }\end{array}$ & 0,300 & $0,072^{* * *}$ & 0,015 & 0,126 & $0,069^{*}$ & 0,049 & 0,045 & 0,074 & 0,018 & 0,091 & 0,070 & 0,036 \\
\hline $\begin{array}{l}\text { Nuevas } \\
\text { actividades } \\
\text { de apoyo }\end{array}$ & 0,186 & $0,058^{\star \star \star}$ & 0,015 & 0,092 & 0,057 & 0,043 & 0,124 & $0,059^{\star *}$ & 0,059 & 0,116 & $0,057^{* *}$ & 0,056 \\
\hline Nuevos bienes & 0,002 & 0,059 & 0,015 & $-0,008$ & 0,057 & $-0,004$ & $-0,043$ & 0,061 & $-0,021$ & 0,061 & 0,058 & 0,030 \\
\hline Nuevos servicios & 0,017 & 0,059 & 0,015 & 0,112 & $0,057^{*}$ & 0,054 & 0,185 & $0,060^{* * *}$ & 0,090 & 0,064 & 0,058 & 0,031 \\
\hline Gasto interno I+D & 0,001 & 0,001 & 0,015 & 0,002 & $0,001^{* *}$ & 0,096 & 0,000 & 0,001 & 0,011 & 0,001 & 0,001 & 0,056 \\
\hline Cooperación & 0,207 & $0,109^{*}$ & 0,015 & 0,237 & $0,107^{\star *}$ & 0,114 & 0,176 & 0,113 & 0,085 & 0,115 & 0,107 & 0,057 \\
\hline Exporta & 0,000 & 0,000 & 0,015 & 0,000 & 0,000 & $-0,013$ & 0,000 & 0,000 & $-0,015$ & 0,000 & $0,000^{*}$ & $-0,046$ \\
\hline $\begin{array}{l}\text { Personal total } \\
\text { en } 1+D\end{array}$ & 0,000 & 0,001 & 0,015 & 0,000 & 0,001 & 0,015 & 0,000 & 0,001 & $-0,012$ & 0,000 & 0,001 & 0,024 \\
\hline $\begin{array}{l}\text { Gasto en } \\
\text { formación }\end{array}$ & $-0,002$ & 0,003 & 0,015 & $-0,002$ & 0,003 & $-0,016$ & 0,000 & 0,003 & $-0,002$ & 0,003 & 0,003 & 0,025 \\
\hline Fondos propios & 0,001 & 0,001 & 0,015 & 0,000 & 0,001 & $-0,019$ & 0,001 & 0,001 & 0,057 & 0,000 & 0,001 & 0,012 \\
\hline /cut1 & $-0,504$ & 0,206 & & 0,333 & 0,202 & & $-1,239$ & 0,215 & & $-0,781$ & 0,203 & \\
\hline /cut2 & 0,033 & 0,205 & & 1,153 & 0,204 & & $-0,466$ & 0,212 & & $-0,030$ & 0,202 & \\
\hline \multirow[t]{2}{*}{ /cut3 } & 1,253 & 0,207 & & 2,261 & 0,207 & & 0,573 & 0,212 & & 1,206 & 0,204 & \\
\hline & $\begin{array}{l}N^{\circ} \\
\text { LR } \\
\text { Log } \\
\text { Prob } \\
\text { Pse }\end{array}$ & $\begin{array}{l}\text { of obs }=1 \\
\text { chi2(22) } \\
\text { lik. }=-195 \\
b>\text { chi2 }= \\
\text { udo } R 2=\end{array}$ & $\begin{array}{l}1642 \\
=86,8 \\
51,57 \\
0,00 \\
0,02\end{array}$ & $\begin{array}{r}N^{\circ} \\
\text { LR c } \\
\text { Log } \\
\text { Prob } \\
\text { Pse } \\
\end{array}$ & $\begin{array}{l}\text { of obs, } 16 \\
\text { hi2 } 2(21)=1 \\
\text { lik, }=-213 \\
>\text { chi } 2= \\
\text { udo } R 2=0,\end{array}$ & $\begin{array}{l}42 \\
03,6 \\
9,07 \\
0,00 \\
024\end{array}$ & $\begin{array}{c}N^{\circ} \text { of ob } \\
\text { LR c } \\
\text { Log } \\
\text { Prob } \\
\text { Pseu }\end{array}$ & $\begin{array}{l}\text { s }=1642 \\
\text { hi2 }(21)=8 \\
\text { lik, }=-180 \\
>\text { chi2 = } \\
\text { do } R 2=0,\end{array}$ & $\begin{array}{l}30,72 \\
9,52 \\
0,00 \\
0218\end{array}$ & $\begin{array}{r}\mathrm{N}^{\circ} \\
\text { LR c } \\
\text { Log } \\
\text { Prob } \\
\text { Psel }\end{array}$ & $\begin{array}{l}\text { of obs }=16 \\
\text { hi2 }(21)=4 \\
\text { liK, }=-207 \\
>\text { chi2 }= \\
\text { udo } R 2=\end{array}$ & $\begin{array}{l}442 \\
19,53 \\
79,6 \\
0,00 \\
0,01\end{array}$ \\
\hline
\end{tabular}

Nota: Estadísticamente significativo al ${ }^{* * *} 99 \%$, ${ }^{* *} 95 \%$ y ${ }^{*} 90 \%$ de confianza.

Fuente: Elaboración propia. 
ción de innovaciones organizacionales puede irse mejorando gradualmente antes de aplicarse de forma extendida a todo el grupo empresarial.

Al igual que en el caso anterior, el tipo de innovación organizacional que genera mayor repercusión sobre la reducción de costes de producción es la introducción de nuevas formas de organización del lugar de trabajo. Mientras que la introducción de nuevos métodos de gestiones del conocimiento y nuevos métodos de gestión de relaciones externas, influyen negativamente sobre la reducción de costes de producción.

La introducción de nuevos servicios y los gastos en I+D a nivel interno tienen una repercusión positiva en la reducción de costes de producción. Teniendo en cuenta que la reducción de costes es una de las motivaciones principales de aquellas empresas que introducen innovaciones tecnológicas, resulta consistente que las innovaciones organizacionales adoptadas refuercen este objetivo. De forma similar, el impacto de las innovaciones organizacionales sobre la reducción de costes, ocurre con mayor probabilidad en las empresas que mantienen acuerdos cooperativos.

En cuanto a las variables que afectan la calidad de los productos elaborados, los resultados mostrados en la tercera columna, las empresas de reciente creación "start-ups" que adoptan innovaciones organizacionales, son capaces de generar un impacto positivo en la calidad de sus productos. Las empresas de reciente creación suelen oponer menor resistencia a los cambios y tener estructuras más flexibles y fáciles de controlar que las empresas con más años de fun- cionamiento. Por otro lado, al tratarse de empresas de reciente creación, la búsqueda de la mejora de la calidad resulta un objetivo prioritario para la consolidación y obtención de mayor cuota de mercado. Al igual que en el resto de las estimaciones la innovación organizacional de tipo 1 genera un efecto negativo sobre la calidad de los productos, mientras que la introducción de innovaciones organizacionales de tipo 2 , repercute positivamente en las mejoras de calidad de los bienes y servicios de la empresa. La introducción de nuevos métodos de fabricación y actividades de apoyo, también repercuten positivamente en las mejoras de calidad obtenidas por las empresas que introducen innovaciones organizacionales.

Finalmente la introducción de nuevos servicios en el mercado también genera una influencia positiva sobre la calidad de los productos ofrecidos. La introducción de nuevos servicios permite la incorporación gradual de mejoras en la oferta de la empresa, con lo cual, se confirma que aquellas empresas capaces de generar innovaciones tecnológicas, se benefician positivamente de la introducción de innovaciones de proceso.

La cuarta columna reporta los resultados correspondientes a la satisfacción de los empleados. En este caso las empresas que obtienen un impacto elevado sobre la satisfacción de sus empleados a través de innovaciones organizacionales, son aquellas empresas exportadoras, que realizan nuevas actividades de apoyo y que introducen innovaciones organizacionales relacionadas con la mejora del lugar de trabajo. Nuevamente las actividades relacionadas con la introducción de nuevos sistemas de gestión del 
Innovaciones organizacionales y su efecto sobre el desempeño empresarial Afcha Chávez, Sergio $M$.

conocimiento, disminuyen la probabilidad de generar un impacto elevado sobre la satisfacción de los empleados.

\section{Conclusiones}

A pesar de ser un tema relevante en la gestión empresarial de las innovaciones, el estudio de las innovaciones organizacionales ha recibido en general poca atención. Sobre todo, destaca la ausencia de evidencia empírica respecto a la adopción de las innovaciones organizacionales y sus efectos sobre el desempeño empresarial.

La falta de una definición ampliamente aceptada y las dificultades en la medición del concepto de innovación organizacional, ha retrasado sin duda la aparición de evidencia empírica respecto a este tipo de innovaciones. La base de datos utilizada en esta investigación (PITEC), permite solventar este problema, ya que aborda explícitamente la medición de las innovaciones organizacionales, utilizando para ello la definición proporcionada en el Manual de Oslo (OCDE, 2005). La integración de esta definición a la encuesta, constituye un avance notable en el seguimiento de las innovaciones no tecnológicas, en tanto que permite generar evidencia empírica comparable, sobre la base de una definición estandarizada, ampliamente aceptada y compartida a nivel internacional.

En un esfuerzo por avanzar en la comprensión de los factores que influyen sobre la adopción de innovaciones organizacionales y el impacto de los distintos tipos de innovación organizacional sobre el desempeño de las empresas, se estimaron un modelo probit trivariante para identificar las variables determinantes en la adopción de tres tipos distintos de IO, y un modelo probit ordenado, para determinar su efecto sobre cuatro indicadores de desempeño de la empresa.

Los resultados del análisis de los determinantes de la adopción de innovaciones muestran los siguientes hallazgos. En primer lugar, la implementación del modelo probit trivariante, destaca la importancia de analizar la incorporación de innovaciones organizacionales como una acción que se lleva a cabo de manera interdependiente. Este hecho, supone que el análisis de dichas innovaciones debe realizarse teniendo en cuenta lo que sucede con el resto de decisiones organizacionales.

En general, las innovaciones organizacionales están positivamente influidas por el tamaño, la introducción de TICs, la adquisición en maquinaria, software y hardware, la cualificación de los recursos humanos disponibles y los acuerdos de cooperación realizados con otras empresas e instituciones científicas como universidades o centros tecnológicos.

Los resultados, muestran que aquellas variables que refuerzan la capacidad de aprendizaje de la organización, influyen significativamente en la adopción de los tres tipos de innovaciones organizacionales. Este resultado, sugiere que la interdependencia entre los distintos tipos de $\mathrm{IO}$, responde a la existencia de elementos que realzan la introducción de innovaciones de forma transversal.

El efecto que cada tipo de innovación organizacional genera, es analizado mediante un modelo probit ordenado. Los resultados de esta estimación, ponen de manifiesto que las innovaciones organi- 
zacionales vinculadas a la organización de los recursos humanos, es la que genera un impacto más importante en los indicadores de desempeño analizados. De esta manera, los indicadores relativos a: tiempos de respuesta de la empresa, costes, calidad de los productos y satisfacción de los trabajadores, se ven afectados positivamente por la adopción de innovaciones organizacionales vinculadas a la organización de la mano de obra de la empresa.

Este trabajo, constituye un punto de partida para continuar avanzando en la investigación de las innovaciones organizacionales. Las futuras líneas de investigación que se plantean, pasan por un análisis temporal más amplio del efecto de las innovaciones organizacionales, así como un estudio más detallado de sus determinantes, que preste atención especial a la introducción de innovaciones en función de la clasificación sectorial.

\section{Referencias bibliográficas}

Afcha, Sergio (2011). Behavioural additionality in the context of regional innovation policy in Spain. Innovation: Management, Policy \& Practice, 13, No. 1, pp. 95-110.

Armbruster, Heidi, Bikfalvi, Andrea, Kinkela, Steffen y Lay Gunter (2008). Organizational innovation: The challenge of measuring non-technical innovation in large-scale surveys. Technovation, 28, No. 10 , pp. 644-657.

Birkinshaw, Julian, Hamel, Gary y Mol, Michael (2008). Management Innovation. Academy of Management Review, 33, No. 4, pp. 825-845.

Black, Sandra y Lynch, Lisa (2004). What's driving the new economy? The bene- fits of workplace innovation. The Economic Journal, 114, No. 493, pp. $97-$ 116.

Cappellari, Lorenzo y Jenkins, Stephen (2006). Calculation of multivariate normal probabilities by simulation, with applications to maximum simulated likelihood estimation. The Stata Journal, 6, No. 2, pp. 156-89.

Caroli, Eve y Jhon Van Reenen (2001). Organization, skills and technology, Evidence from a panel of british and french establishments. Quarterly Journal of Economics, 116, No. 4, pp. 1449-92.

Chang, Su-Chao y Lee, Ming-Shing (2008). The linkage between knowledge accumulation capability and organizational innovation. Journal of Knowledge Management 12, 1, pp.3-20.

Cohen, Wesley y Levinthal, Daniel (1990). Absorptive capacity: New perspective on learning and innovation'. Administrative Science Quarterly, 35, No.1, pp. 128-52.

Daft, Richard (1978), A dual-core model of organizational innovation. The academy of management journal, 21, No. 2, pp. 193-210.

Damanpour, Fariborz y Evan, Williams (1984). Organizational innovation and performance, The problem of organizational lag. Administrative science quarterly, 29, No. 3, pp. 392-409.

Damanpour, Fariborz, Walker Richard y Avellaneda, Claudia (2009). Combinative effects of innovation types and organizational performance, A longitudinal study of service organizations. Journal of Management Studies, 46, No. 4, pp. 650-75.

EUROSTAT (2005). Task Force Meeting on Oslo Manual Revision. Draft of the Third Edition, Luxembourg.

Fariñas, José y Lopez, Alberto (2011). Medición de las innovaciones organizati- 
Innovaciones organizacionales y su efecto sobre el desempeño empresarial Afcha Chávez, Sergio $M$.

vas y efectos sobre la productividad: Evidencias sobre la empresa española. Papeles de Economía Española, № 127 , pp. 120-139.

FECYT (2011). La base de datos Panel de Innovación Tecnológica (PITEC). FECYT. Madrid. Consultado el 23/09/2011, y disponible en: http://icono.fecyt.es/05\%29Publi/AA\%29panel/base datosPITEC_Mayo_2011_esp.pdf

Frambach, Ruud y Schillewaert, Niels (2002). Organizational innovation adoption. A multi-level framework of determinants and opportunities for future research. Journal of Business Research, 55, No. 2, pp 163-76.

Gera, Surendra y Gu, Wulong (2004). The effect of organizational innovation and information technology on firm performance. International Performance Monitor, 9, pp. 37-51.

Geroski, Paul (1995). Innovation and Competitive Advantage. OECD Economics Department Working Papers. Paris, Francia.

Goldman, Steven, Nagel, Roger y Preiss, Kenneth (1995), Agile competitors and virtual organizations. Strategies for enriching the customer. Vol. 414, New York, USA. Van Nostrand Reinhold.

Greenan, Nathalie (2003). Organizational change, technology, employment and skills. An empirical study of french manufacturing. Cambridge Journal of Economics, 27, No. 2, p. 287.

Hammer, Michael y Champy, James (1995). Reengineering the corporation, A manifesto for business revolution. London, UK. Nicholas Brealey.

Jansen, Justins, Van den Bosch, Frans y Volberda, Henk (2006). Exploratory innovation, exploitative innovation, and performance: Effects of organizational antecedents and environmental moderators. Management Science, 52, No. 11, pp. 1661-74.

Lam, Alice (2004). Organizational innovation. MPRA Paper.

Laursen, Keld y Salter, Amon (2004). Searching high and low: what types of firms use universities as a source of innovation?. Research Policy 33, No. 8, pp. 1201-1215.

Long, Scott y Freese, Jeremy (2001). Regression models for categorical dependent variables using stata. Texas, Usa. Stata Press College Station.

Lynch, Lisa (2007). The adoption and diffusion of organizational innovation. Evidence for the us economy. National Bureau of Economic Research Working Paper, Cambridge, Mass., USA.

Miranda, Alfonso y Rabe-Hesketh, Sofia (2006). Maximum likelihood estimation of endogenous switching and sample selection models for binary, ordinal, and count variables. Stata Journal, 6, No. 3, pp 285-308.

OCDE (2005). Oslo Manual Guidelines for Collecting and Interpreting Innovation Data. Paris, OECD Publishing.

Pfeffer, Jeffrey y Salancik, Gerald (2003). The External Control of Organizations: A Resource Dependence Perspective, Harper \& Row. New York, USA

Piva, Maria Cristina, Santarelli, Enrico y Vivarelli, Marco (2006). Technological and organizational changes as determinants of the skill bias, Evidence from the italian machinery industry. Managerial and Decision Economics, 27, No. 1, pp. 63-73.

Polder, Michael, Van Leeuwen, George, Mohnen, Pierre y Raymond, Wladimir (2010). Product, process and organizational innovation: Drivers, complementarity and productivity effects. 
University of Mastricht, 23719, MPRA.

Roberts, Peters y Amit, Raffi (2003). The dynamics of innovative activity and competitive advantage: The case of Australian retail banking, 1981 to 1995. Organization Science, 14, No. 2, pp. 107-22.

Rosenberg, Nathan (1993). Dentro de la Caja Negra: Tecnologia y Economia. Barcelona, España, La llar del llibre.
Subramanian, Ashok y Nilakanta, Sree (1996). Organizational innovativeness. Exploring the relationship between organizational determinants of innovation, types of innovations, and measures of organizational performance. Omega, 24, No. 6, pp. 631-47.

Womack, James, Jones, Daniel y Roos, Daniel (1990). The machine that changed the world. New York, USA. Rawson Associates. 